\title{
Adaptive control of Mittag-Leffler stabilization and synchronization for delayed fractional-order BAM neural networks
}

\author{
Weike Cheng ${ }^{1}$, Ailong Wu² ${ }^{2}$, Jin-E Zhang ${ }^{2}$ and Biwen Li ${ }^{2}$
}

${ }^{\text {*Correspondence: }}$ hbnuwu@yeah.net
${ }^{2}$ College of Mathematics and
Statistics, Hubei Normal University,
Huangshi, China
Full list of author information is
available at the end of the article

available at the end of the article

\begin{abstract}
This paper is committed to studying adaptive control design of Mittag-Leffler stabilization and synchronization for delayed fractional-order bidirectional associative memory (BAM) neural networks. Considering better dynamic property and steady state performance of the system, we adopt adaptive control approaches to stabilize and synchronize two types of delayed fractional-order BAM neural network. It is a remarkable fact, based on adaptive control scheme, the method of auxiliary functions, Mittag-Leffler stabilization and synchronization theories with respect to fractional-order systems, the adaptive controllers are very well designed in a controlled system and two coupled systems, separately. Two examples are performed to illustrate the advantage of the presented theoretic analysis and results.
\end{abstract}

Keywords: Fractional-order systems; BAM neural networks; Time delays; Mittag-Leffler stabilization and synchronization; Adaptive control design

\section{Introduction}

Recently, the rapid development of fractional calculus has shown that it is an attractive hot research topic, and fractional calculus has been used successfully in different scientific and technological fields $[1,2]$. For all we know, the generalized form of ordinary integerorder calculus is said to be the fractional calculus, which originated in the 17th century [3], at that time, fractional calculus could not be well studied in light of the lack of comprehensive and systematic theoretical knowledge. Along with the depth of understanding of the fractional calculus, researchers found that fractional calculus can not only provide a mathematical modeling tool, but also describe the complex dynamic behavior that ordinary integer-order calculus cannot. Namely, a fractional-order system has more efficient and accurate capabilities than conventional integer-order system and is closer to the realworld cases. In many practical dynamic processes, a fractional-order system also has the advantage of many degrees of freedom and the ability of general computation. Additionally, the infinite memory characteristics of a fractional-order system, which can include the genetic information of the past, are displayed incisively and vividly. It is precisely because of these excellent performances that the application and research of fractional-order systems in many areas has developed well $[4,5]$. However, a material fact worth noting is that a fractional-order system cannot directly use the control strategies of conventional

(c) The Author(s) 2019. This article is distributed under the terms of the Creative Commons Attribution 4.0 International License (http://creativecommons.org/licenses/by/4.0/), which permits unrestricted use, distribution, and reproduction in any medium, provided you give appropriate credit to the original author(s) and the source, provide a link to the Creative Commons license, and indicate if changes were made. 
integer-order system due to the complexity and particularity of the fractional-order system. As a consequence, how to choose effective tools and reasonable schemes to control a fractional-order system is worthy of consideration.

In 1987, Kosko first put forward bidirectional associative memory (BAM) neural networks with double layers [6], which revealed the intimate connection between the neurons arranged in the U-layer and the neurons arranged in the V-layer. In general, the neurons of the U-layer are fully integrated with the neurons of the V-layer, meanwhile the neurons of the U-layer and the V-layer have no interconnection. Because of this special performance of double-layer networks, their numerous applications have shown interest and importance in the areas of artificial intelligence, combinatorial optimization, signal processing, and image processing $[7,8]$. In addition, as we all know, in the process of signal transmission between neurons on double-layer networks, some hereditary characteristics are embodied in intelligent information processing of the two layers. Based on this important discovery, it is very essential and significant that BAM neural networks are incorporated into infinite memory terms. From the point of view of fractional-order neurodynamic systems, unlike the locality of integer-order derivative, fractional-order derivative with singularity and nonlocality has infinite memory and hereditary characteristics of diverse processes with potential ability. Hence, the analysis and synthesis with respect to fractional-order BAM neural networks is worth looking into.

Whether it is electronic or biological network, there exists a general phenomenon called time delay [9]. In the transmission of signals between neurons, due to the finite speed of propagation and the limited switching speed on the amplifier, it is inevitable that the problem with time delays is always encountered. Particularly, for neural networks, there are many parallel paths with different length and size of the nodes, because complex networks have the features of time and space, single and coupled networks with time delays cannot be generalized. That is, the time delays of complex networks are not the same due to different distributions of propagation delays. According to the degree of correlation between time delay and time, time delay can be divided into constant delay [10] and time-varying delay [11]. Although there exists constant delay in some networks, time delay changes with time in general. For the time-varying delayed networks, the stability of networks is not only influenced, but even the dynamic behaviors that occur in networks may be complex and difficult to handle. In other words, the existence of time delays would lead to a destruction of the networks dynamics, it can be seen that time delays have an important influence on the dynamic characteristics of networks. So compared to the networks without time delays, it is significant and necessary to introduce the phenomenon of time delays with more abundant dynamic properties into neural networks. Up to now, all kinds of literature sources on delayed neural networks have attracted a lot of experimental and theoretical attention $[12,13]$. However, significant results for time-varying delayed fractional-order systems are seldom reported. Thus, it is worthy to investigate a challenging problem regarding time delays in fractional-order systems.

Stabilization and synchronization are common and typical phenomena in the real world. Over the last few decades, stabilization and synchronization problems of complex control systems made an enormous difference in the domains of physics, engineering, and science $[14,15]$. Just as its name implies, the meaning of stabilization is that a controlled system is stable by applying an external force. That is, given an uncontrolled system of arbitrary states, this uncontrolled system can reach a stable state mainly because the external con- 
troller applied to the controlled system plays a significant role. Unlike the stabilization of a single network, synchronization usually occurs in two or more networks. Theoretically, in order to realize synchronization, there must be some variable coupling between the systems, and as time goes by, the trajectories of coupled systems become increasingly consistent. In short, synchronization could be construed as the tracking of target trajectory. Among numerous synchronization phenomena, drive-response synchronization is the most common type of synchronization, which is widely used in various networks [16]. To sum up, it can be said that both stabilization and synchronization are an indispensable part of system dynamics. Particularly, in fractional-order systems, some attractive results regarding stabilization and synchronization phenomena have been introduced [17, 18]. From another point of view, talking about fractional-order systems, we know that MittagLeffler function is one of the important functions, and related properties of Mittag-Leffler are often studied [19]. However, Mittag-Leffler stabilization and synchronization are litthe investigated in double-layer systems. Considering this fact, it is worthwhile studying Mittag-Leffler stabilization and synchronization that can get some results of small gains.

For control theory, it is widely known that adaptive control design has become an explored area of mathematical studies, and adaptive control is often used in many practical applications in recent years due to the fact that adaptive control itself has better stable performance and higher tracking accuracy [20]. Generally speaking, adaptive control can be regarded as a feedback control that has the ability to change its performance characteristics in response to its environment, so that the system can work in the best condition according to predefined criteria. The difference from the classic feedback control is that adaptive control is based on less prior knowledge about the model. That is to say, adaptive control needs to extract the relevant information about the model constantly in the running process of the system, then the model will be gradually improved and more closer to the real situation. As far as we know, the parameters of the traditional feedback controller are fixed, when the internal properties and external disturbances of the system change a lot, the performance of the system can be degraded and even become unstable. The emergence of adaptive control effectively solves these problems, for example, adaptive control is applied to aircraft systems with high requirements, so good performance over a wide range of the altitudes and speeds of the airplane can be maintained. With the development of adaptive control, some novel results have been shown in the relevant literature $[21,22]$. It should be noted that adaptive control is much more complicated than the classic feedback control, how to achieve the ultimate purpose of optimum control effect is a challenging task. As a consequence, constructing highly-efficient, explored and analyzed adaptive control is worth thinking about.

With the above discussion, we try to use adaptive control as a kind of better control scheme to explore Mittag-Leffler stabilization and synchronization on two types of delayed fractional-order BAM neural network. For purpose of the realization with MittagLeffler stabilization and synchronization in double-layer networks, the adaptive controllers with system and adjustable parameters are designed. Moreover, on the basis of essentially more general adaptive control conditions, the corresponding results on MittagLeffler stabilization and synchronization are obtained. Roughly stated, our main contributions of the paper are summed up into two points: (1) It is the first time that adaptive control design is applied to delayed fractional-order BAM neural networks. (2) Several Mittag-Leffler stabilization and synchronization criteria are first put forward in two 
types of delayed fractional-order BAM neural network on the basis of adaptive control scheme. There is no doubt that adaptive control strategy can be more targeted and efficient to improve the convergence character of fractional-order dynamics, and adaptive control scheme combined with Gronwall-like inequality with generalized type can be conveniently applied in a wider range of application areas. Actually, adaptive control approach only needs to set adjustable parameter values, so that it can be a powerful analysis tool for high-performance systems. Besides, adaptive control design of other fractional-order systems can be extended based on the obtained criteria. It is hoped that the theoretical analysis in this paper can provide the heuristic results regarding adaptive control scheme for fractional-order control theory.

This paper has the following four parts to constitute an overall analytical framework. In Sect. 2, the relevant preliminaries are stated from the three aspects, namely: fractional calculus, model description, definitions and properties. Several sufficient criteria are proposed to realize Mittag-Leffler stabilization and synchronization by adaptive control approaches in Sect. 3. Section 4 is utilized to give two examples to demonstrate the efficiency of our theoretic analysis. In the end, Sect. 5 summarizes the general conclusions.

\section{Preliminaries}

\subsection{Fractional calculus}

To facilitate the description of our model, two basic notions for fractional calculus are recalled.

The Caputo derivative of order $q>0$ of a function $\mathscr{H}(t) \in C^{n+1}\left(\left[t_{0},+\infty\right), \mathcal{R}\right)$ is characterized as

$$
{ }_{t_{0}}^{C} D_{t}^{q} \mathcal{H}(t)=\frac{1}{\Gamma(n-q)} \int_{t_{0}}^{t} \frac{\mathscr{H}^{(n)}(s)}{(t-s)^{q-n+1}} \mathrm{~d} s
$$

where $t \geq t_{0}, n$ denotes a positive integer satisfying $n-1<q<n$, and $\Gamma(\cdot)$ stands for the Gamma function.

Mittag-Leffler function $E_{q}(\cdot)$ with one-parameter is given as

$$
E_{q}(s)=\sum_{k=0}^{+\infty} \frac{s^{k}}{\Gamma(k q+1)}
$$

where $q>0, s$ represents a complex number, and $\Gamma(\cdot)$ stands for the Gamma function.

Remark 2.1 Fractional calculus occupies a very important position in control science and engineering. Particularly, the Caputo derivative has been the most frequently mentioned fractional-order operator for neurodynamic systems, mainly on account of the fact that the initial conditions with the Caputo derivative and the initial conditions with the integerorder derivative have much in common, and the Caputo derivative has more representations based on the descriptions of real-world situations.

Remark 2.2 It is worth noting that there are essential differences between the convergence property of traditional integer-order systems and that of fractional-order systems. For integer-order systems, we know that the exponential function can be utilized to deal with the dynamics problems. For fractional-order systems, the exponential function is no 
longer applicable because fractional-order systems possess abnormal and complex convergence behaviors. So the exponential function can be replaced by Mittag-Leffler function in fractional-order systems, which is a generally used function that can be widely applied to different types of factional-order system.

\subsection{Model description}

Throughout the paper, $\mathcal{R}^{n}$ stands for the $n$-dimensional real space; given a real vector $\alpha=\left(\alpha_{1}, \alpha_{2}, \ldots, \alpha_{n}\right)^{T} \in \mathcal{R}^{n}$, the norm is recorded as $\|\alpha\|=\sum_{i=1}^{n}\left|\alpha_{i}\right|$.

We focus on the following delayed fractional-order BAM neural networks of the form

$$
\begin{aligned}
& { }_{t_{0}}^{C} D_{t}^{q} x_{i}(t)=-\beta_{i} x_{i}(t)+\sum_{j=1}^{m} a_{i j} f_{j}\left(y_{j}(t)\right)+\sum_{j=1}^{m} b_{i j} f_{j}\left(y_{j}\left(t-\sigma_{j}(t)\right)\right)+p_{i}(t), \\
& { }_{t_{0}}^{C} D_{t}^{q} y_{j}(t)=-\gamma_{j} y_{j}(t)+\sum_{i=1}^{n} c_{j i} g_{i}\left(x_{i}(t)\right)+\sum_{i=1}^{n} d_{j i} g_{i}\left(x_{i}\left(t-\theta_{i}(t)\right)\right)+r_{j}(t),
\end{aligned}
$$

where $t \geq t_{0}, i=1,2, \ldots, n, j=1,2, \ldots, m, 0<q<1, x_{i}(t)$ and $y_{j}(t)$ mean the state variables, $\beta_{i}>0$ and $\gamma_{j}>0$ represent the self-inhibition, $a_{i j}, b_{i j}, c_{j i}$ and $d_{j i}$ stand for the synaptic strengths, $p_{i}(t)$ and $r_{j}(t)$ denote the external inputs, the time delays $\sigma_{j}(t)$ and $\theta_{i}(t)$ are continuous and bounded on $[0,+\infty)$, the output functions $f_{j}(\cdot), g_{i}(\cdot)$ satisfy $f_{j}(0)=0, g_{i}(0)=0$ and $\forall v, \bar{v} \in \mathcal{R}$,

$$
\begin{aligned}
& \left|f_{j}(v)-f_{j}(\bar{v})\right| \leq k_{j}|v-\bar{v}|, \\
& \left|g_{i}(v)-g_{i}(\bar{v})\right| \leq h_{i}|v-\bar{v}|,
\end{aligned}
$$

in which $k_{j}>0, h_{i}>0(i=1,2, \ldots, n, j=1,2, \ldots, m)$.

The initial conditions regarding system (1) are described by

$$
x_{i}\left(t_{0}+s\right)=\psi_{i}(s), \quad y_{j}\left(t_{0}+s\right)=\varphi_{j}(s), \quad s \in[-\tau, 0],
$$

for $i=1,2, \ldots, n, j=1,2, \ldots, m$, where $\tau=\max _{1 \leq i \leq n, 1 \leq j \leq m}\left\{\sigma_{j}(t), \theta_{i}(t)\right\}$, real-valued functions $\psi_{i}(s)$ and $\varphi_{j}(s)$ defined on $[-\tau, 0]$ are continuous, and the corresponding norms are expressed as

$$
\|\psi\|=\sum_{i=1}^{n} \sup _{s \in[-\tau, 0]}\left\{\left|\psi_{i}(s)\right|\right\}, \quad\|\varphi\|=\sum_{j=1}^{m} \sup _{s \in[-\tau, 0]}\left\{\left|\varphi_{j}(s)\right|\right\} .
$$

For Mittag-Leffler synchronization, we focus on another type of system (1) as the drive system governed by

$$
\begin{aligned}
& { }_{t_{0}}^{C} D_{t}^{q} x_{i}(t)=-\beta_{i} x_{i}(t)+\sum_{j=1}^{m} a_{i j} f_{j}\left(y_{j}(t)\right)+\sum_{j=1}^{m} b_{i j} f_{j}\left(y_{j}\left(t-\sigma_{j}(t)\right)\right), \\
& { }_{t_{0}}^{C} D_{t}^{q} y_{j}(t)=-\gamma_{j} y_{j}(t)+\sum_{i=1}^{n} c_{j i} g_{i}\left(x_{i}(t)\right)+\sum_{i=1}^{n} d_{j i} g_{i}\left(x_{i}\left(t-\theta_{i}(t)\right)\right),
\end{aligned}
$$


in which $t \geq t_{0}, i=1,2, \ldots, n, j=1,2, \ldots, m$, where the initial conditions of system (6) are the same as described for system (1).

We aim to design the following associated response system:

$$
\begin{aligned}
& { }_{t_{0}}^{C} D_{t}^{q} \tilde{x}_{i}(t)=-\beta_{i} \tilde{x}_{i}(t)+\sum_{j=1}^{m} a_{i j} f_{j}\left(\tilde{y}_{j}(t)\right)+\sum_{j=1}^{m} b_{i j} f_{j}\left(\tilde{y}_{j}\left(t-\sigma_{j}(t)\right)\right)+p_{i}(t), \\
& { }_{t_{0}}^{C} D_{t}^{q} \tilde{y}_{j}(t)=-\gamma_{j} \tilde{y}_{j}(t)+\sum_{i=1}^{n} c_{j i} g_{i}\left(\tilde{x}_{i}(t)\right)+\sum_{i=1}^{n} d_{j i} g_{i}\left(\tilde{x}_{i}\left(t-\theta_{i}(t)\right)\right)+r_{j}(t),
\end{aligned}
$$

in which $t \geq t_{0}, i=1,2, \ldots, n, j=1,2, \ldots, m$, and $p_{i}(t), r_{j}(t)$ signify the control inputs that need to be constructed appropriately.

The initial conditions for system (7) are given by

$$
\tilde{x}_{i}\left(t_{0}+s\right)=\tilde{\psi}_{i}(s), \quad \tilde{y}_{j}\left(t_{0}+s\right)=\tilde{\varphi}_{j}(s), \quad s \in[-\tau, 0],
$$

for $i=1,2, \ldots, n, j=1,2, \ldots, m$, where $\tau=\max _{1 \leq i \leq n, 1 \leq j \leq m}\left\{\sigma_{j}(t), \theta_{i}(t)\right\}$, real-valued functions $\tilde{\psi}_{i}(s)$ and $\tilde{\varphi}_{j}(s)$ defined on $[-\tau, 0]$ are continuous, and the corresponding norms are expressed as

$$
\|\tilde{\psi}\|=\sum_{i=1}^{n} \sup _{s \in[-\tau, 0]}\left\{\left|\tilde{\psi}_{i}(s)\right|\right\}, \quad\|\tilde{\varphi}\|=\sum_{j=1}^{m} \sup _{s \in[-\tau, 0]}\left\{\left|\tilde{\varphi}_{j}(s)\right|\right\} .
$$

Let $z_{i}(t)=\tilde{x}_{i}(t)-x_{i}(t)$ for $i=1,2, \ldots, n$ and $\tilde{z}_{j}(t)=\tilde{y}_{j}(t)-y_{j}(t)$ for $j=1,2, \ldots, m$, then the error system between (6) and (7) is obtained by

$$
\begin{aligned}
& { }_{t_{0}}^{C} D_{t}^{q} z_{i}(t)=-\beta_{i} z_{i}(t)+\sum_{j=1}^{m} a_{i j} f_{j}\left(\tilde{z}_{j}(t)\right)+\sum_{j=1}^{m} b_{i j} f_{j}\left(\tilde{z}_{j}\left(t-\sigma_{j}(t)\right)\right)+p_{i}(t), \\
& { }_{t_{0}}^{C} D_{t}^{q} \tilde{z}_{j}(t)=-\gamma_{j} \tilde{z}_{j}(t)+\sum_{i=1}^{n} c_{j i} g_{i}\left(z_{i}(t)\right)+\sum_{i=1}^{n} d_{j i} g_{i}\left(z_{i}\left(t-\theta_{i}(t)\right)\right)+r_{j}(t),
\end{aligned}
$$

where $t \geq t_{0}, i=1,2, \ldots, n, j=1,2, \ldots, m, f_{j}\left(\tilde{z}_{j}(t)\right)=f_{j}\left(\tilde{y}_{j}(t)\right)-f_{j}\left(y_{j}(t)\right), g_{i}\left(z_{i}(t)\right)=g_{i}\left(\tilde{x}_{i}(t)\right)-$ $g_{i}\left(x_{i}(t)\right)$, and $p_{i}(t), r_{j}(t)$ signify the control inputs that need to be constructed appropriately. Naturally, the initial conditions for system (10) can be stated by

$$
z_{i}\left(t_{0}+s\right)=\tilde{\psi}_{i}(s)-\psi_{i}(s), \quad \tilde{z}_{j}\left(t_{0}+s\right)=\tilde{\varphi}_{j}(s)-\varphi_{j}(s), \quad s \in[-\tau, 0]
$$

for $i=1,2, \ldots, n, j=1,2, \ldots, m$, where $\tau=\max _{1 \leq i \leq n, 1 \leq j \leq m}\left\{\sigma_{j}(t), \theta_{i}(t)\right\}$, real-valued functions $\tilde{\psi}_{i}(s)-\psi_{i}(s)$ and $\tilde{\varphi}_{j}(s)-\varphi_{j}(s)$ defined on $[-\tau, 0]$ are continuous, and the corresponding norms are expressed as

$$
\begin{aligned}
& \|\tilde{\psi}-\psi\|=\sum_{i=1}^{n} \sup _{s \in[-\tau, 0]}\left\{\left|\tilde{\psi}_{i}(s)-\psi_{i}(s)\right|\right\}, \\
& \|\tilde{\varphi}-\varphi\|=\sum_{j=1}^{m} \sup _{s \in[-\tau, 0]}\left\{\left|\tilde{\varphi}_{j}(s)-\varphi_{j}(s)\right|\right\} .
\end{aligned}
$$


Remark 2.3 In the process of our model description, we adopt drive-response systems (6) and (7) to realize Mittag-Leffler synchronization. The drive system (6) can be called a master system, which is uncontrolled due to lack of control inputs. The response system (7) is also said to be a slave system, which possesses control inputs. In theory, after a long enough period of time, the error system (10) can realize Mittag-Leffler stability based on some appropriate feedback control design, so the coupled systems (6) and (7) are referred to be Mittag-Leffler synchronized.

\subsection{Definitions and properties}

In this subsection, in order to better analyze the problems of Mittag-Leffler dynamics, some important definitions about Mittag-Leffler dynamics and several relevant lemmas are provided, which are utilized for our theoretical results later.

Definition 2.1 For any $\eta>0$, if there exist $T>0, \mathcal{M}(\eta)>0$ and $\delta>0$ satisfying for any $t \geq t_{0}+T$

$$
\|x(t)\|+\|y(t)\| \leq \mathcal{M}(\eta) E_{q}\left(-\delta\left(t-t_{0}\right)^{q}\right)
$$

when $\|\psi\|+\|\varphi\| \leq \eta$, then the zero solution of system (1) is called Mittag-Leffler stabilizable, where $p_{i}(t)=0, r_{j}(t)=0(i=1,2, \ldots, n, j=1,2, \ldots, m)$.

Remark 2.4 What needs to be pointed out is that the original reference in Definition 2.1 is from the definition of Mittag-Leffler synchronization for fractional-order single-layer networks, which has been given in [2, Definition 4.2]. In view of the structural property of double-layer networks and the connection between stability and synchronization, it is not a difficult to see that the definition of Mittag-Leffler dynamics on single-layer networks is extended to double-layer networks, so there is no doubt that Definition 2.1 can be obtained.

Remark 2.5 From Definition 2.1, we can learn that the sum of two final values will converge to zero after a fixed amount of time, that is, the system can realize ultimate Mittag-Leffler stability as time $t$ goes to infinity. In addition, $\delta$ is also said to be the degree of MittagLeffler stability.

Definition 2.2 If the controlled system of (1) realizes Mittag-Leffler stability based on some appropriate feedback control design, then system (1) is called Mittag-Leffler stabilizable.

Definition 2.3 If the error dynamics system (10) realizes Mittag-Leffler stability, then the drive system (6) and the response system (7) are called Mittag-Leffler synchronizable.

Remark 2.6 It is easy to see that the definitions with respect to Mittag-Leffler stabilization and synchronization are based on the stability definition of Mittag-Leffler. And it is also important to notice that Mittag-Leffler dynamics involves asymptotic behavior due to the properties of Mittag-Leffler function, namely, Mittag-Leffler stability means asymptotic 
stability, the same goes for Mittag-Leffler stabilization and Mittag-Leffler synchronization.

Lemma 2.1 ([1]) Let $0<q<1$ be given. If $\mathscr{H}(t) \in C^{1}\left[t_{0},+\infty\right)$, then

$$
{ }_{t_{0}}^{C} D_{t}^{q}|\mathcal{H}(t)| \leq \operatorname{sgn}(\mathscr{H}(t))_{t_{0}}^{C} D_{t}^{q} \mathcal{H}(t)
$$

for $t \geq t_{0}$, where

$$
{ }_{t_{0}}^{C} D_{t}^{q}|\mathcal{H}(t)|=\frac{1}{\Gamma(1-q)} \int_{t_{0}}^{t} \frac{\frac{\mathrm{d}}{\mathrm{d} s}|\mathcal{H}(s)|}{(t-s)^{q}} \mathrm{~d} s .
$$

Remark 2.7 Taking into account the complexity of Caputo derivative of the absolute value function in fractional calculus, Lemma 2.1 gives the connection between the Caputo derivative of function $|\mathscr{H}(t)|$ and Caputo derivative of function $\mathscr{H}(t)$, which provides a convenient approach for fractional calculation.

Lemma $2.2([2])$ If $\mathfrak{W}(t)$ and $U(t)$ are two continuous nonnegative functions satisfying

$$
{ }_{t_{0}}^{C} D_{t}^{q}(\mathcal{W}(t)+\mathcal{U}(t)) \leq-\delta \mathcal{W}(t)
$$

where $0<q<1, \delta>0$, then there exists a constant $T>0$ such that

$$
\mathcal{W}(t) \leq\left(\mathfrak{W}\left(t_{0}\right)+\mathcal{U}\left(t_{0}\right)+\ell\right) E_{q}\left(-\delta\left(t-t_{0}\right)^{q}\right)
$$

for any positive constant $\ell$, where $t \geq t_{0}+T$.

Remark 2.8 In order to ensure that Mittag-Leffler stabilization and synchronization can be realized, Lemma 2.2 offers the convergence property regarding Gronwall-like inequality of generalized type, which can deal with the problems of convergence very well in many types of fractional-order systems, and it can be utilized to establish a criterion with respect to fractional-order dynamics. Moreover, more relevant details about Lemma 2.2 can be found in [2, Lemma 4.3].

\section{Main results}

After a detailed introduction and explanation, in this section, we present adaptive control strategies, which can be used to realize Mittag-Leffler stabilization and synchronization in the corresponding systems.

For the sake of narrative, the adaptive control schemes are first used to deal with MittagLeffler stabilization and synchronization problems in double-layer networks, and then the theoretical results are reviewed and analyzed. 


\subsection{Mittag-Leffler stabilization}

Theorem 3.1 Suppose positive constants $\mu_{i}, \breve{\mu}_{i}, \xi_{j}, \breve{\xi}_{j}$ are arbitrary. If system (1) is controlled based on the following adaptive control scheme:

$$
\left\{\begin{array}{l}
p_{i}(t)=-w_{i}(t) x_{i}\left(t-\theta_{i}(t)\right)-\breve{w}_{i}(t) x_{i}(t), \\
r_{j}(t)=-u_{j}(t) y_{j}\left(t-\sigma_{j}(t)\right)-\breve{u}_{j}(t) y_{j}(t), \\
{ }_{t_{0}}^{C} D_{t}^{q} w_{i}(t)=\mu_{i}\left|x_{i}\left(t-\theta_{i}(t)\right)\right|, \\
{ }_{t_{0}}^{C} D_{t}^{q} u_{j}(t)=\xi_{j}\left|y_{j}\left(t-\sigma_{j}(t)\right)\right|, \\
{ }_{t_{0}}^{C} D_{t}^{q} \breve{w}_{i}(t)=\breve{\mu}_{i}\left|x_{i}(t)\right| \\
{ }_{t_{0}}^{C} D_{t}^{q} \breve{u}_{j}(t)=\breve{\xi}_{j}\left|y_{j}(t)\right|
\end{array}\right.
$$

in which $i=1,2, \ldots, n, j=1,2, \ldots, m$, then Mittag-Leffler stabilization can be realized for system (1).

Proof Under the controller (13), the controlled system (1) is rewritten as

$$
\begin{aligned}
{ }_{t_{0}}^{C} D_{t}^{q} x_{i}(t)= & -\beta_{i} x_{i}(t)+\sum_{j=1}^{m} a_{i j} f_{j}\left(y_{j}(t)\right)+\sum_{j=1}^{m} b_{i j} f_{j}\left(y_{j}\left(t-\sigma_{j}(t)\right)\right) \\
& -w_{i}(t) x_{i}\left(t-\theta_{i}(t)\right)-\breve{w}_{i}(t) x_{i}(t), \\
{ }_{t_{0}}^{C} D_{t}^{q} y_{j}(t)= & -\gamma_{j} y_{j}(t)+\sum_{i=1}^{n} c_{j i} g_{i}\left(x_{i}(t)\right)+\sum_{i=1}^{n} d_{j i} g_{i}\left(x_{i}\left(t-\theta_{i}(t)\right)\right) \\
& -u_{j}(t) y_{j}\left(t-\sigma_{j}(t)\right)-\breve{u}_{j}(t) y_{j}(t),
\end{aligned}
$$

where $t \geq t_{0}, i=1,2, \ldots, n, j=1,2, \ldots, m$.

We construct an auxiliary function

$$
W(t)=\sum_{i=1}^{n}\left|x_{i}(t)\right|+\sum_{j=1}^{m}\left|y_{j}(t)\right| .
$$

Through Lemma 2.1, by (14) we can derive

$$
\begin{aligned}
{ }_{t_{0}}^{C} D_{t}^{q} W(t) \leq & \sum_{i=1}^{n} \operatorname{sgn}\left(x_{i}(t)\right)_{t_{0}}^{C} D_{t}^{q} x_{i}(t)+\sum_{j=1}^{m} \operatorname{sgn}\left(y_{j}(t)\right)_{t_{0}}^{C} D_{t}^{q} y_{j}(t) \\
\leq & \sum_{i=1}^{n}\left[-\beta_{i}\left|x_{i}(t)\right|+\sum_{j=1}^{m}\left|a_{i j}\right|\left|f_{j}\left(y_{j}(t)\right)\right|+\sum_{j=1}^{m}\left|b_{i j}\right|\left|f_{j}\left(y_{j}\left(t-\sigma_{j}(t)\right)\right)\right|\right. \\
& \left.-w_{i}(t)\left|x_{i}\left(t-\theta_{i}(t)\right)\right|-\breve{w}_{i}(t)\left|x_{i}(t)\right|\right]+\sum_{j=1}^{m}\left[-\gamma_{j}\left|y_{j}(t)\right|\right. \\
& +\sum_{i=1}^{n}\left|c_{j i}\right|\left|g_{i}\left(x_{i}(t)\right)\right|+\sum_{i=1}^{n}\left|d_{j i}\right|\left|g_{i}\left(x_{i}\left(t-\theta_{i}(t)\right)\right)\right| \\
& \left.-u_{j}(t)\left|y_{j}\left(t-\sigma_{j}(t)\right)\right|-\breve{u}_{j}(t)\left|y_{j}(t)\right|\right]
\end{aligned}
$$




$$
\begin{aligned}
\leq & \sum_{i=1}^{n}\left[-\beta_{i}\left|x_{i}(t)\right|+\sum_{j=1}^{m}\left|a_{i j}\right| k_{j}\left|y_{j}(t)\right|+\sum_{j=1}^{m}\left|b_{i j}\right| k_{j}\left|y_{j}\left(t-\sigma_{j}(t)\right)\right|\right. \\
& \left.-w_{i}(t)\left|x_{i}\left(t-\theta_{i}(t)\right)\right|-\breve{w}_{i}(t)\left|x_{i}(t)\right|\right]+\sum_{j=1}^{m}\left[-\gamma_{j}\left|y_{j}(t)\right|\right. \\
& +\sum_{i=1}^{n}\left|c_{j i}\right| h_{i}\left|x_{i}(t)\right|+\sum_{i=1}^{n}\left|d_{j i}\right| h_{i}\left|x_{i}\left(t-\theta_{i}(t)\right)\right| \\
& \left.-u_{j}(t)\left|y_{j}\left(t-\sigma_{j}(t)\right)\right|-\breve{u}_{j}(t)\left|y_{j}(t)\right|\right] \\
\leq & \sum_{i=1}^{n}\left[-\beta_{i}\left|x_{i}(t)\right|+\sum_{j=1}^{m}\left|c_{j i}\right| h_{i}\left|x_{i}(t)\right|-\breve{w}_{i}(t)\left|x_{i}(t)\right|\right. \\
& \left.+\sum_{j=1}^{m}\left|d_{j i}\right| h_{i}\left|x_{i}\left(t-\theta_{i}(t)\right)\right|-w_{i}(t)\left|x_{i}\left(t-\theta_{i}(t)\right)\right|\right] \\
& +\sum_{j=1}^{m}\left[-\gamma_{j}\left|y_{j}(t)\right|+\sum_{i=1}^{n}\left|a_{i j}\right| k_{j}\left|y_{j}(t)\right|-\breve{u}_{j}(t)\left|y_{j}(t)\right|\right. \\
& \left.+\sum_{i=1}^{n}\left|b_{i j}\right| k_{j}\left|y_{j}\left(t-\sigma_{j}(t)\right)\right|-u_{j}(t)\left|y_{j}\left(t-\sigma_{j}(t)\right)\right|\right] .
\end{aligned}
$$

In the following, consider another auxiliary function

$$
\begin{aligned}
U(t)= & \sum_{i=1}^{n}\left[\frac{1}{2 \mu_{i}}\left(w_{i}(t)-w_{i}^{\Delta}\right)^{2}+\frac{1}{2 \breve{\mu}_{i}}\left(\breve{w}_{i}(t)-\breve{w}_{i}^{\Delta}\right)^{2}\right] \\
& +\sum_{j=1}^{m}\left[\frac{1}{2 \xi_{j}}\left(u_{j}(t)-u_{j}^{\Delta}\right)^{2}+\frac{1}{2 \breve{\xi}_{j}}\left(\breve{u}_{j}(t)-\breve{u}_{j}^{\Delta}\right)^{2}\right] .
\end{aligned}
$$

By (16) and (17), it holds

$$
\begin{aligned}
{ }_{t_{0}}^{C} D_{t}^{q}(W(t)+U(t)) \leq & \sum_{i=1}^{n}\left[-\beta_{i}\left|x_{i}(t)\right|+\sum_{j=1}^{m}\left|c_{j i}\right| h_{i}\left|x_{i}(t)\right|-\breve{w}_{i}^{\Delta}\left|x_{i}(t)\right|\right. \\
& \left.+\sum_{j=1}^{m}\left|d_{j i}\right| h_{i}\left|x_{i}\left(t-\theta_{i}(t)\right)\right|-w_{i}^{\Delta}\left|x_{i}\left(t-\theta_{i}(t)\right)\right|\right] \\
& +\sum_{j=1}^{m}\left[-\gamma_{j}\left|y_{j}(t)\right|+\sum_{i=1}^{n}\left|a_{i j}\right| k_{j}\left|y_{j}(t)\right|-\breve{u}_{j}^{\Delta}\left|y_{j}(t)\right|\right. \\
& \left.+\sum_{i=1}^{n}\left|b_{i j}\right| k_{j}\left|y_{j}\left(t-\sigma_{j}(t)\right)\right|-u_{j}^{\Delta}\left|y_{j}\left(t-\sigma_{j}(t)\right)\right|\right] .
\end{aligned}
$$

Selecting $\breve{w}_{i}^{\Delta}, w_{i}^{\Delta}, \breve{u}_{j}^{\Delta}$ and $u_{j}^{\Delta}$ large enough to satisfy

$$
\left\{\begin{array}{l}
\breve{w}_{i}^{\Delta} \geq-\beta_{i}+\sum_{j=1}^{m}\left|c_{j i}\right| h_{i}+\delta_{1}, \\
\breve{u}_{j}^{\Delta} \geq-\gamma_{j}+\sum_{i=1}^{n}\left|a_{i j}\right| k_{j}+\delta_{2}, \\
w_{i}^{\Delta} \geq \sum_{j=1}^{m}\left|d_{j i}\right| h_{i} \\
u_{j}^{\Delta} \geq \sum_{i=1}^{n}\left|b_{i j}\right| k_{j}
\end{array}\right.
$$


where $\delta_{1}$ and $\delta_{2}$ are positive constants, we get

$$
{ }_{t_{0}}^{C} D_{t}^{q}(W(t)+U(t)) \leq-\delta W(t)
$$

where $\delta=\min \left\{\delta_{1}, \delta_{2}\right\}$.

According to Lemma 2.2, it follows from (20) that for any positive constant $\ell$ there exists $T \geq 0$ such that

$$
\begin{aligned}
\|x(t)\|+\|y(t)\| & \leq\left(W\left(t_{0}\right)+U\left(t_{0}\right)+\ell\right) E_{q}\left(-\delta\left(t-t_{0}\right)^{q}\right) \\
& \leq M(\eta) E_{q}\left(-\delta\left(t-t_{0}\right)^{q}\right),
\end{aligned}
$$

for any $t \geq t_{0}+T$, when $\|\psi\|+\|\varphi\| \leq \eta$, where

$$
\begin{aligned}
M(\eta)= & \eta+\ell+\sum_{i=1}^{n}\left[\frac{1}{2 \mu_{i}}\left(w_{i}\left(t_{0}\right)-w_{i}^{\Delta}\right)^{2}+\frac{1}{2 \breve{\mu}_{i}}\left(\breve{w}_{i}\left(t_{0}\right)-\breve{w}_{i}^{\Delta}\right)^{2}\right] \\
& +\sum_{j=1}^{m}\left[\frac{1}{2 \xi_{j}}\left(u_{j}\left(t_{0}\right)-u_{j}^{\Delta}\right)^{2}+\frac{1}{2 \breve{\xi}_{j}}\left(\breve{u}_{j}\left(t_{0}\right)-\breve{u}_{j}^{\Delta}\right)^{2}\right] .
\end{aligned}
$$

Hence, under the adaptive controller (13), it can be claimed that system (1) realizes Mittag-Leffler stabilization.

\subsection{Mittag-Leffler synchronization}

Theorem 3.2 Suppose positive constants $\mu_{i}, \breve{\mu}_{i}, \xi_{j}, \breve{\xi}_{j}$ are arbitrary. If the response system (7) is controlled based on the following adaptive control scheme:

$$
\left\{\begin{array}{l}
p_{i}(t)=-w_{i}(t) z_{i}\left(t-\theta_{i}(t)\right)-\breve{w}_{i}(t) z_{i}(t), \\
r_{j}(t)=-u_{j}(t) \tilde{z}_{j}\left(t-\sigma_{j}(t)\right)-\breve{u}_{j}(t) \tilde{z}_{j}(t), \\
{ }_{t_{0}}^{C} D_{t}^{q} w_{i}(t)=\mu_{i}\left|z_{i}\left(t-\theta_{i}(t)\right)\right|, \\
{ }_{t_{0}}^{C} D_{t}^{q} u_{j}(t)=\xi_{j}\left|\tilde{z}_{j}\left(t-\sigma_{j}(t)\right)\right|, \\
{ }_{t_{0}}^{C} D_{t}^{q} \breve{w}_{i}(t)=\breve{\mu}_{i}\left|z_{i}(t)\right| \\
{ }_{t_{0}}^{C} D_{t}^{q} \breve{u}_{j}(t)=\breve{\xi}_{j}\left|\tilde{z}_{j}(t)\right|
\end{array}\right.
$$

in which $i=1,2, \ldots, n, j=1,2, \ldots, m$, then Mittag-Leffler synchronization can be realized for the drive system (6) and response system (7).

Proof By the controller (21), the error system (10) is rewritten as

$$
\begin{aligned}
{ }_{t_{0}}^{C} D_{t}^{q} z_{i}(t)= & -\beta_{i} z_{i}(t)+\sum_{j=1}^{m} a_{i j} f_{j}\left(\tilde{z}_{j}(t)\right)+\sum_{j=1}^{m} b_{i j} f_{j}\left(\tilde{z}_{j}\left(t-\sigma_{j}(t)\right)\right) \\
& -w_{i}(t) z_{i}\left(t-\theta_{i}(t)\right)-\breve{w}_{i}(t) z_{i}(t), \\
{ }_{t_{0}}^{C} D_{t}^{q} \tilde{z}_{j}(t)= & -\gamma_{j} \tilde{z}_{j}(t)+\sum_{i=1}^{n} c_{j i} g_{i}\left(z_{i}(t)\right)+\sum_{i=1}^{n} d_{j i} g_{i}\left(z_{i}\left(t-\theta_{i}(t)\right)\right) \\
& -u_{j}(t) \tilde{z}_{j}\left(t-\sigma_{j}(t)\right)-\breve{u}_{j}(t) \tilde{z}_{j}(t),
\end{aligned}
$$

where $t \geq t_{0}, i=1,2, \ldots, n, j=1,2, \ldots, m$. 
We design an auxiliary function

$$
W(t)=\sum_{i=1}^{n}\left|z_{i}(t)\right|+\sum_{j=1}^{m}\left|\tilde{z}_{j}(t)\right| .
$$

Through Lemma 2.1, by (23) we can derive

$$
\begin{aligned}
& { }_{t_{0}}^{C} D_{t}^{q} W(t) \leq \sum_{i=1}^{n} \operatorname{sgn}\left(z_{i}(t)\right)_{t_{0}}^{C} D_{t}^{q} z_{i}(t)+\sum_{j=1}^{m} \operatorname{sgn}\left(\tilde{z}_{j}(t)\right)_{t_{0}}^{C} D_{t}^{q} \tilde{z}_{j}(t) \\
& \leq \sum_{i=1}^{n}\left[-\beta_{i}\left|z_{i}(t)\right|+\sum_{j=1}^{m}\left|a_{i j}\right|\left|f_{j}\left(\tilde{z}_{j}(t)\right)\right|+\sum_{j=1}^{m}\left|b_{i j}\right|\left|f_{j}\left(\tilde{z}_{j}\left(t-\sigma_{j}(t)\right)\right)\right|\right. \\
& \left.-w_{i}(t)\left|z_{i}\left(t-\theta_{i}(t)\right)\right|-\breve{w}_{i}(t)\left|z_{i}(t)\right|\right]+\sum_{j=1}^{m}\left[-\gamma_{j}\left|\tilde{z}_{j}(t)\right|\right. \\
& +\sum_{i=1}^{n}\left|c_{j i}\right|\left|g_{i}\left(z_{i}(t)\right)\right|+\sum_{i=1}^{n}\left|d_{j i}\right|\left|g_{i}\left(z_{i}\left(t-\theta_{i}(t)\right)\right)\right| \\
& \left.-u_{j}(t)\left|\tilde{z}_{j}\left(t-\sigma_{j}(t)\right)\right|-\breve{u}_{j}(t)\left|\tilde{z}_{j}(t)\right|\right] \\
& \leq \sum_{i=1}^{n}\left[-\beta_{i}\left|z_{i}(t)\right|+\sum_{j=1}^{m}\left|a_{i j}\right| k_{j}\left|\tilde{z}_{j}(t)\right|+\sum_{j=1}^{m}\left|b_{i j}\right| k_{j}\left|\tilde{z}_{j}\left(t-\sigma_{j}(t)\right)\right|\right. \\
& \left.-w_{i}(t)\left|z_{i}\left(t-\theta_{i}(t)\right)\right|-\breve{w}_{i}(t)\left|z_{i}(t)\right|\right]+\sum_{j=1}^{m}\left[-\gamma_{j}\left|\tilde{z}_{j}(t)\right|\right. \\
& +\sum_{i=1}^{n}\left|c_{j i}\right| h_{i}\left|z_{i}(t)\right|+\sum_{i=1}^{n}\left|d_{j i}\right| h_{i}\left|z_{i}\left(t-\theta_{i}(t)\right)\right| \\
& \left.-u_{j}(t)\left|\tilde{z}_{j}\left(t-\sigma_{j}(t)\right)\right|-\breve{u}_{j}(t)\left|\tilde{z}_{j}(t)\right|\right] \\
& \leq \sum_{i=1}^{n}\left[-\beta_{i}\left|z_{i}(t)\right|+\sum_{j=1}^{m}\left|c_{j i}\right| h_{i}\left|z_{i}(t)\right|-\breve{w}_{i}(t)\left|z_{i}(t)\right|\right. \\
& \left.+\sum_{j=1}^{m}\left|d_{j i}\right| h_{i}\left|z_{i}\left(t-\theta_{i}(t)\right)\right|-w_{i}(t)\left|z_{i}\left(t-\theta_{i}(t)\right)\right|\right] \\
& +\sum_{j=1}^{m}\left[-\gamma_{j}\left|\tilde{z}_{j}(t)\right|+\sum_{i=1}^{n}\left|a_{i j}\right| k_{j}\left|\tilde{z}_{j}(t)\right|-\breve{u}_{j}(t)\left|\tilde{z}_{j}(t)\right|\right. \\
& \left.+\sum_{i=1}^{n}\left|b_{i j}\right| k_{j}\left|\tilde{z}_{j}\left(t-\sigma_{j}(t)\right)\right|-u_{j}(t)\left|\tilde{z}_{j}\left(t-\sigma_{j}(t)\right)\right|\right] .
\end{aligned}
$$

In the following, consider another auxiliary function

$$
\begin{aligned}
U(t)= & \sum_{i=1}^{n}\left[\frac{1}{2 \mu_{i}}\left(w_{i}(t)-w_{i}^{\Delta}\right)^{2}+\frac{1}{2 \breve{\mu}_{i}}\left(\breve{w}_{i}(t)-\breve{w}_{i}^{\Delta}\right)^{2}\right] \\
& +\sum_{j=1}^{m}\left[\frac{1}{2 \xi_{j}}\left(u_{j}(t)-u_{j}^{\Delta}\right)^{2}+\frac{1}{2 \breve{\xi}_{j}}\left(\breve{u}_{j}(t)-\breve{u}_{j}^{\Delta}\right)^{2}\right] .
\end{aligned}
$$


By (24) and (25), it holds

$$
\begin{aligned}
{ }_{t_{0}}^{C} D_{t}^{q}(W(t)+U(t)) \leq & \sum_{i=1}^{n}\left[-\beta_{i}\left|z_{i}(t)\right|+\sum_{j=1}^{m}\left|c_{j i}\right| h_{i}\left|z_{i}(t)\right|-\breve{w}_{i}^{\Delta}\left|z_{i}(t)\right|\right. \\
& \left.+\sum_{j=1}^{m}\left|d_{j i}\right| h_{i}\left|z_{i}\left(t-\theta_{i}(t)\right)\right|-w_{i}^{\Delta}\left|z_{i}\left(t-\theta_{i}(t)\right)\right|\right] \\
& +\sum_{j=1}^{m}\left[-\gamma_{j}\left|\tilde{z}_{j}(t)\right|+\sum_{i=1}^{n}\left|a_{i j}\right| k_{j}\left|\tilde{z}_{j}(t)\right|-\breve{u}_{j}^{\Delta}\left|\tilde{z}_{j}(t)\right|\right. \\
& \left.+\sum_{i=1}^{n}\left|b_{i j}\right| k_{j}\left|\tilde{z}_{j}\left(t-\sigma_{j}(t)\right)\right|-u_{j}^{\Delta}\left|\tilde{z}_{j}\left(t-\sigma_{j}(t)\right)\right|\right] .
\end{aligned}
$$

Selecting $\breve{w}_{i}^{\Delta}, w_{i}^{\Delta}, \breve{u}_{j}^{\Delta}$ and $u_{j}^{\Delta}$ large enough to satisfy

$$
\left\{\begin{array}{l}
\breve{w}_{i}^{\Delta} \geq-\beta_{i}+\sum_{j=1}^{m}\left|c_{j i}\right| h_{i}+\delta_{1}, \\
\breve{u}_{j}^{\Delta} \geq-\gamma_{j}+\sum_{i=1}^{n}\left|a_{i j}\right| k_{j}+\delta_{2}, \\
w_{i}^{\Delta} \geq \sum_{j=1}^{m}\left|d_{j i}\right| h_{i}, \\
u_{j}^{\Delta} \geq \sum_{i=1}^{n}\left|b_{i j}\right| k_{j},
\end{array}\right.
$$

where $\delta_{1}$ and $\delta_{2}$ are positive constants, we get

$$
{ }_{t_{0}}^{C} D_{t}^{q}(W(t)+U(t)) \leq-\delta W(t),
$$

where $\delta=\min \left\{\delta_{1}, \delta_{2}\right\}$.

According to Lemma 2.2, it follows from (28) that for any positive constant $\ell$ there exists $T \geq 0$ such that

$$
\begin{aligned}
\|z(t)\|+\|\tilde{z}(t)\| & \leq\left(W\left(t_{0}\right)+U\left(t_{0}\right)+\ell\right) E_{q}\left(-\delta\left(t-t_{0}\right)^{q}\right) \\
& \leq M(\eta) E_{q}\left(-\delta\left(t-t_{0}\right)^{q}\right),
\end{aligned}
$$

for any $t \geq t_{0}+T$, when $\|\tilde{\psi}-\psi\|+\|\tilde{\varphi}-\varphi\| \leq \eta$, where

$$
\begin{aligned}
M(\eta)= & \eta+\ell+\sum_{i=1}^{n}\left[\frac{1}{2 \mu_{i}}\left(w_{i}\left(t_{0}\right)-w_{i}^{\Delta}\right)^{2}+\frac{1}{2 \breve{\mu}_{i}}\left(\breve{w}_{i}\left(t_{0}\right)-\breve{w}_{i}^{\Delta}\right)^{2}\right] \\
& +\sum_{j=1}^{m}\left[\frac{1}{2 \xi_{j}}\left(u_{j}\left(t_{0}\right)-u_{j}^{\Delta}\right)^{2}+\frac{1}{2 \breve{\xi}_{j}}\left(\breve{u}_{j}\left(t_{0}\right)-\breve{u}_{j}^{\Delta}\right)^{2}\right] .
\end{aligned}
$$

Hence, under the adaptive controller (21), it can be claimed that the drive system (6) and response system (7) realize Mittag-Leffler synchronization.

Remark 3.1 As we have seen, the auxiliary functions (15), (17), (23), and (25) are actually Lyapunov-like functions. Taking auxiliary functions (15) and (23) for example, this form of auxiliary functions are chosen because they make full use of the properties of two variables in BAM networks, so it is natural to go back to the considered systems (1) and 
(10). Additionally, the construction of auxiliary functions (17) and (25) is very ingenious, it is easy to find that " 2 " in the denominator and " 2 " in the index just cancel each other out when taking the derivatives of auxiliary functions (17) and (25), the results are not affected and the calculation is much simper.

Remark 3.2 In the course of our derivation, a Caputo fractional-order derivative can be calculated in two ways for the auxiliary function $W(t)$ in (15). The first method is to use the definition of Caputo fractional-order derivative directly, and then Lemma 2.1 is utilized to simplify results. The second method is that auxiliary function $W(t)$ can be treated as two functions, and then Lemma 2.1 is utilized for each function. No matter which approach, the Caputo fractional-order derivative of $W(t)$ can be shown to satisfy ${ }_{t_{0}}^{C} D_{t}^{q} W(t) \leq \sum_{i=1}^{n} \operatorname{sgn}\left(x_{i}(t)\right)_{t_{0}}^{C} D_{t}^{q} x_{i}(t)+\sum_{j=1}^{m} \operatorname{sgn}\left(y_{j}(t)\right)_{t_{0}}^{C} D_{t}^{q} y_{j}(t)$. Similarly, these two methods are used to calculate the Caputo fractional-order derivative for the auxiliary function $W(t)$ in (23).

Remark 3.3 As introduced in Theorems 3.1 and 3.2, it is shown that there are similarities and differences in the analysis of Mittag-Leffler stabilization and synchronization. For the method of auxiliary functions, we can find that the auxiliary functions constructed with respect to Mittag-Leffler stabilization or synchronization are similar. In addition, for Mittag-Leffler stabilization, system (1) itself is controlled based on a suitable adaptive controller, and the realization of stabilization for system (1) implies that system (14) must realize Mittag-Leffler stability. For Mittag-Leffler synchronization, the response system (7) is controlled based on a suitable adaptive controller, and the realization of synchronization for the drive-response systems (6) and (7) implies that the error system (22) must realize Mittag-Leffler stability.

Remark 3.4 It is clear that the adaptive control schemes are very effective for dealing with Mittag-Leffler dynamics problems of fractional-order systems in Theorems 3.1 and 3.2. There is no doubt that the key-point of the proposed control method is the application of a family of fractional-order nonlinear systems. Through the transformation of auxiliary functions, the function terms of the adaptive control design are eliminated, and then the results of Mittag-Leffler dynamics can be obtained by using Lemma 2.2. In fact, Lemma 2.2 becomes the principal ingredient in the proof process which brings together the adaptive control and Mittag-Leffler dynamics. That is, if there exists a lemma which can find a bridge between the adaptive control and other dynamics in fractional-order systems, then the results regarding other dynamics can be derived. It follows that the main idea of the designed adaptive control can be used widely due to the flexibility. Hence, there is generality in this adaptive control approach for fractional-order systems.

Remark 3.5 What is noteworthy is that the change of adaptive control gains $\mu_{i}, \breve{\mu}_{i}, \xi_{j}$, $\breve{\xi}_{j}$ will affect the change of control inputs under adaptive control strategies (13) and (21), and meanwhile, the stabilization and synchronization speeds will also change accordingly. Hence, when adaptive control strategy is utilized for fractional-order dynamics, the adaptive control gains $\mu_{i}, \breve{\mu}_{i}, \xi_{j}, \breve{\xi}_{j}$ can be selected according to the requirements of designer, such as appropriate control inputs and fast convergence speed. 
Remark 3.6 In the contemporary literature, from the perspective of the control design, there are many new results about the network control systems; for example, see [23, 24]. However, in fractional-order systems, the adaptive control strategy based on double-layer networks has not been studied, yet; so Theorems 3.1 and 3.2 are new. Comparing with similar findings in [2], the obtained results in this paper generalize the cases on single-layer networks and add a meaningful criterion regarding Mittag-Leffler stabilization, which reveals the differences and connections between Mittag-Leffler stabilization and synchronization and has certain representativeness.

Remark 3.7 Looking through the whole process of analysis, there is no doubt that the prominent role of design schemes with respect to adaptive controllers cannot be ignored. Besides, according to the characteristics of adjustable parameters, adaptive control is more advantageous for complex systems, such as the controlled system with unknown features or large disturbances. Namely, it is an interesting issue that adaptive control is applied to more systems in future studies.

\section{Illustrative examples}

In this section, for the purpose of substantiating the validity of the above theoretical criteria, two numerical examples are put forward through computer simulation.

Example 4.1 We focus on a class of delayed fractional-order BAM neural networks

$$
\begin{aligned}
& { }_{t_{0}}^{C} D_{t}^{q} x(t)=-0.11 x(t)+0.15 f(y(t))+0.12 f(y(t-\sigma(t)))+p(t), \\
& { }_{t_{0}}^{C} D_{t}^{q} y(t)=-0.13 y(t)-0.14 g(x(t))-0.16 g(x(t-\theta(t)))+r(t),
\end{aligned}
$$

where $t \geq 0, q=0.96, f(\cdot)=g(\cdot)=\tanh (\cdot), \sigma(t)=1, \theta(t)=0.9$, then $\tau=1$.

Under system (29) without adaptive controller, the time response curves of system (29) are depicted in Fig. 1, which shows that state trajectories cannot convergence to the origin.

Taking $\mu=0.01, \breve{\mu}=0.01, \xi=0.01, \breve{\xi}=0.01$ and setting initial values as $w(0)=0.1$, $\breve{w}(0)=0.1, u(0)=0.25, \breve{u}(0)=0.05$, the time evolution of stabilization curves of system (29) with initial conditions $x(s)=1, y(s)=-2$ for $s \in[-1,0]$ is portrayed in Fig. 2 . The adaptive control gains $w(t), \breve{w}(t), u(t), \breve{u}(t)$ are described in Figs. 3 and 4 , respectively.

Figure 1 Time response curves of system (29) without adaptive controller

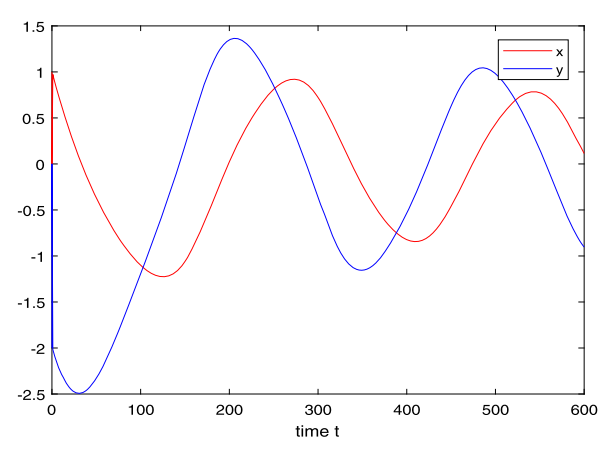




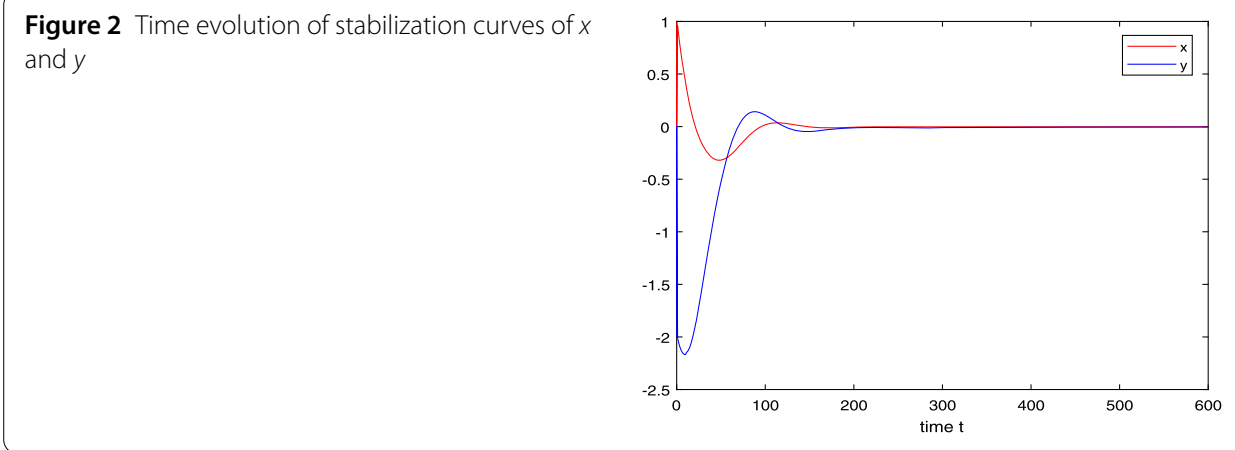

Figure 3 Time evolution of the adaptive control gains $w(t), \breve{w}(t)$

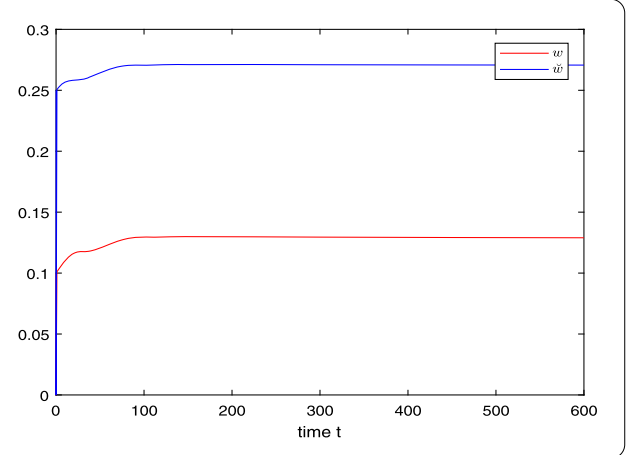

Figure 4 Time evolution of the adaptive control gains $u(t), \breve{u}(t)$

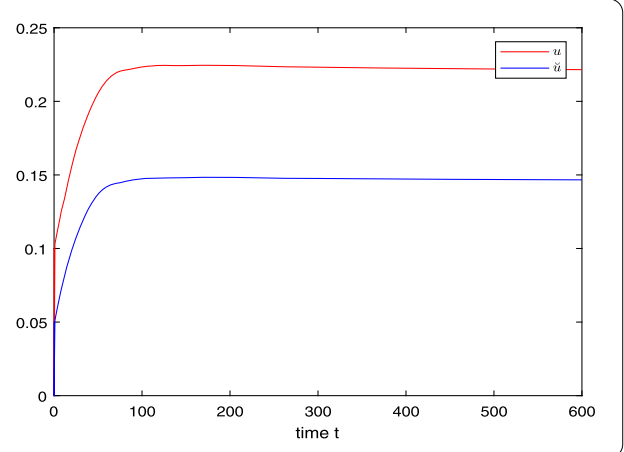

Example 4.2 We focus on the following type of delayed fractional-order BAM neural network as a drive system:

$$
\begin{aligned}
& { }_{t_{0}}^{C} D_{t}^{q} x(t)=-1.2 x(t)+1.8 f(y(t))+1.3 f(y(t-\sigma(t))), \\
& { }_{t_{0}}^{C} D_{t}^{q} y(t)=-1.4 y(t)-1.6 g(x(t))-1.1 g(x(t-\theta(t))),
\end{aligned}
$$

where $t \geq 0, q=0.96, f(\cdot)=g(\cdot)=\sin (\cdot), \sigma(t)=1, \theta(t)=0.9$, then $\tau=1$.

The computer simulation of system (30) starting from initial conditions $x(s)=4, y(s)=$ -5 for $s \in[-1,0]$ is depicted in Fig. 5, which displays a chaotic attractor in system (30).

The associated response system is designed by

$$
\begin{aligned}
& { }_{t_{0}}^{C} D_{t}^{q} \tilde{x}(t)=-1.2 \tilde{x}(t)+1.8 f(\tilde{y}(t))+1.3 f(\tilde{y}(t-\sigma(t)))+p(t), \\
& { }_{t_{0}}^{C} D_{t}^{q} \tilde{y}(t)=-1.4 \tilde{y}(t)-1.6 g(\tilde{x}(t))-1.1 g(\tilde{x}(t-\theta(t)))+r(t),
\end{aligned}
$$

where $t \geq 0, q=0.96, f(\cdot)=g(\cdot)=\sin (\cdot), \sigma(t)=1, \theta(t)=0.9$, and $\tau=1$. 
Figure 5 Chaotic attractor of system (30)

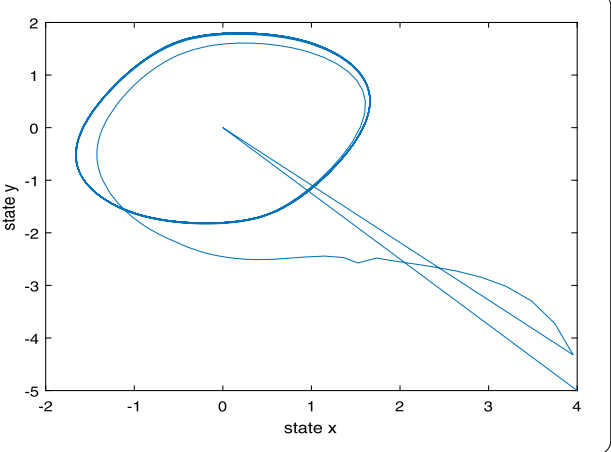

Figure 6 Time evolution of synchronization curves of $x$ and $\tilde{x}$

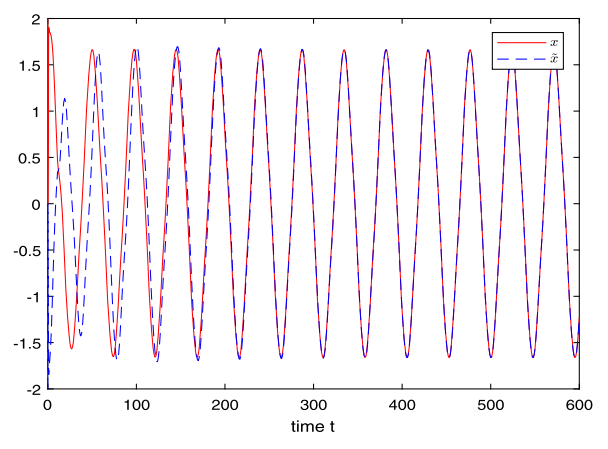

Figure 7 Time evolution of synchronization curves of $y$ and $\tilde{y}$

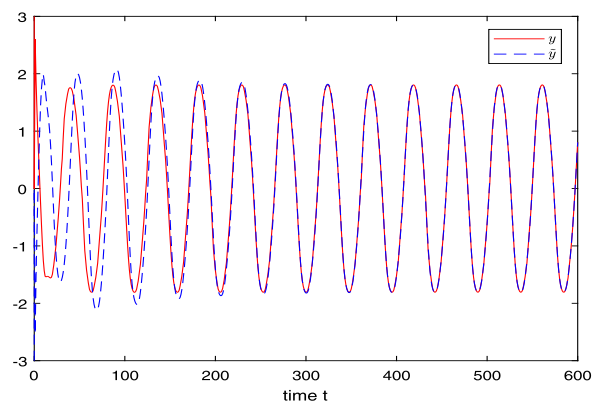

Figure 8 Time evolution of synchronization error of $z$

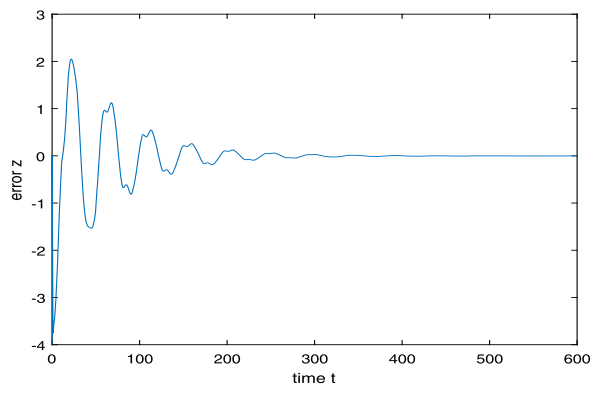

Taking $\mu=0.01, \breve{\mu}=0.01, \xi=0.01, \breve{\xi}=0.01$ and setting initial values as $w(0)=0.05$, $\breve{w}(0)=0.2, u(0)=0.25, \breve{u}(0)=0.2$, Figs. 6-9 portray the time evolution regarding adaptive synchronization curves and adaptive synchronization errors of systems (30) and (31) 


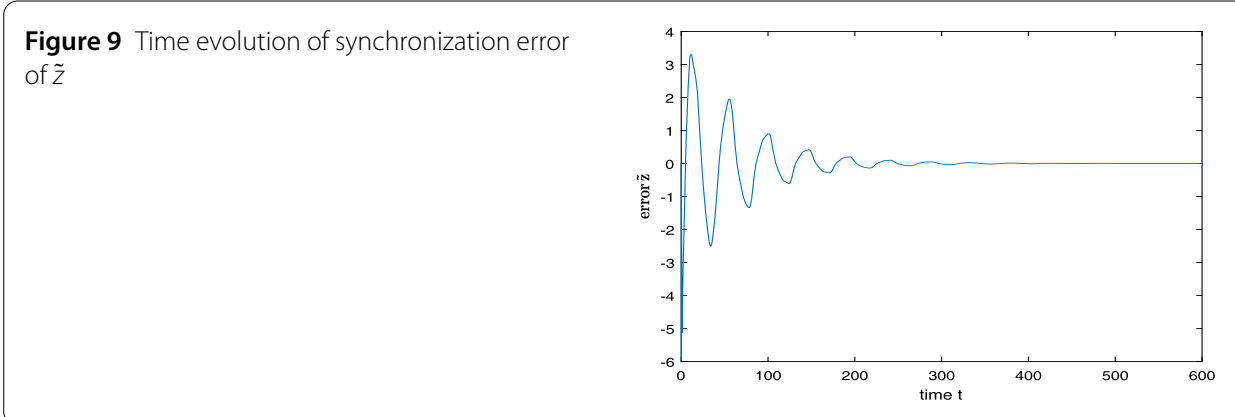

Figure 10 Time evolution of the adaptive control gains $w(t), \breve{w}(t)$

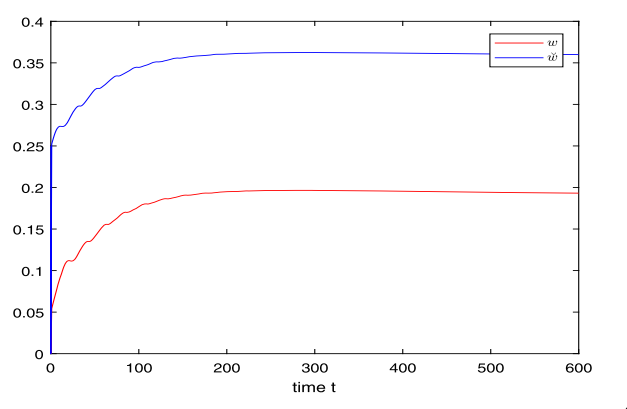

Figure 11 Time evolution of the adaptive control gains $u(t), \breve{u}(t)$

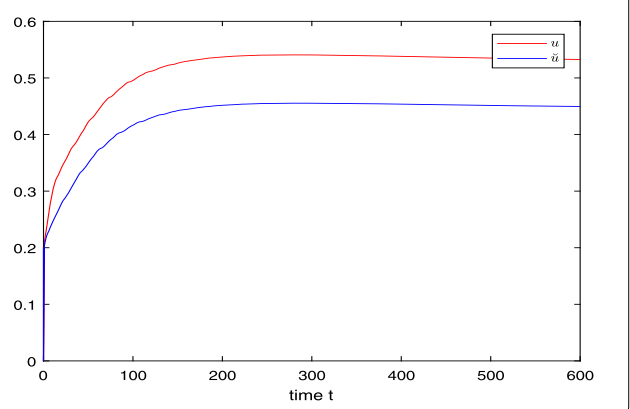

starting from initial values $x(s)=2, y(s)=3, \tilde{x}(s)=-2, \tilde{y}(s)=-3$ for $s \in[-1,0]$, respectively. Figures 10 and 11 depict the adaptive control gains $w(t), \breve{w}(t), u(t), \breve{u}(t)$, respectively.

Remark 4.1 Comparing with the numerical simulation results of Mittag-Leffler stabilization and synchronization, the object of stabilization and the object of synchronization that converge to the origin are different. For Mittag-Leffler stabilization, the curves of two states $x$ and $y$ converge to the origin, while for Mittag-Leffler synchronization, the curves of two errors $z$ and $\tilde{z}$ converge to the origin. Additionally, Figs. 3, 4, 10, and 11 show that the adaptive controllers are very well designed.

\section{Conclusion}

The research of dynamic behavior of fractional-order systems by various control schemes has attracted extensive attention, nevertheless, the analysis and design of a kind of doublelayer network by adaptive control approach has seldom been studied. In this paper, for a class of delayed fractional-order BAM neural networks, we use adaptive control strategy to exploit Mittag-Leffler stabilization and synchronization. Our main results of the paper are 
that adaptive controllers with adjustable parameters are constructed to stabilize and synchronize two types of delayed double-layer network. Based on the adaptive control design, the method of auxiliary functions and Mittag-Leffler dynamics theory of fractional-order systems, several sufficient criteria guaranteeing the realization of Mittag-Leffler stabilization in a controlled system and the realization of Mittag-Leffler synchronization in two coupled systems are shown. Moreover, the corresponding numerical simulations show the validity of the proposed results.

It should be noted that the adaptive controllers only contain some adjustable parameters and a little priori information about system itself. As the future work, much room for improvement remains based on adaptive control strategy, the corresponding adaptive controllers can be designed appropriately according to the requirements of different fractional-order systems. Indeed, those issues may be considerable research topics in the future.

\section{Funding}

This work was supported in part by the Natural Science Foundation of China under Grant 61773152, in part by the Natural Science Foundation of Hubei Province of China under Grant 2018 CFB532.

\section{Competing interests}

The authors declare that they have no competing interests.

\section{Authors' contributions}

All authors contributed equally to this work. All authors read and approved the final manuscript.

\section{Author details}

${ }^{1}$ College of Applied Mathematics, Hubei Normal University, Huangshi, China. ${ }^{2}$ College of Mathematics and Statistics, Hubei Normal University, Huangshi, China.

\section{Publisher's Note}

Springer Nature remains neutral with regard to jurisdictional claims in published maps and institutional affiliations.

Received: 15 April 2019 Accepted: 31 July 2019 Published online: 13 August 2019

\section{References}

1. Chen, B.S., Chen, J.J.: Global asymptotical $\omega$-periodicity of a fractional-order non-autonomous neural networks. Neural Netw. 68, 78-88 (2015)

2. Chen, J.J., Chen, B.S., Zeng, Z.G.: $O\left(t^{-\alpha}\right)$-Synchronization and Mittag-Leffler synchronization for the fractional-orde memristive neural networks with delays and discontinuous neuron activations. Neural Netw. 100, 10-24 (2018)

3. Samko, S.G., Kilbas, A.A., Marichev, O.I.: Fractional Integrals and Derivatives and Some of Their Applications. Nauka i Technika, Minsk (1987)

4. Zhang, J.E.: Centralized and decentralized data-sampling principles for outer-synchronization of fractional-order neural networks. Complexity 2017, Article ID 6290646 (2017)

5. Wu, A.L., Liu, L., Huang, T.W., Zeng, Z.G.: Mittag-Leffler stability of fractional-order neural networks in the presence of generalized piecewise constant arguments. Neural Netw. 85, 118-127 (2017)

6. Kosko, B.: Adaptive bidirectional associative memories. Appl. Opt. 26(23), 4947-4960 (1987)

7. Wei, X.R., Qiu, Z.P.: Anti-periodic solutions for BAM neural networks with time delays. Appl. Math. Comput. 221, 221-229 (2013)

8. Cao, J.D., Wan, Y.: Matrix measure strategies for stability and synchronization of inertial BAM neural network with time delays. Neural Netw. 53, 165-172 (2014)

9. Chen, L.P., Wu, R.C., Cao, J.D., Liu, J.B.: Stability and synchronization of memristor-based fractional-order delayed neural networks. Neural Netw. 71, 37-44 (2015)

10. Yang, X.J., Song, Q.K., Liu, Y.R., Zhao, Z.J.: Finite-time stability analysis of fractional-order neural networks with delay, Neurocomputing 152, 19-26 (2015)

11. Aouiti, C., Gharbia, I.B., Cao, J.D., M'hamdi, M.S., Alsaedi, A.: Existence and global exponential stability of pseudo almost periodic solution for neutral delay BAM neural networks with time-varying delay in leakage terms. Chaos Solitons Fractals 107, 111-127 (2018)

12. Huang, C.D., Nie, X.B., Zhao, X., Song, Q.K., Tu, Z.W., Xiao, M., Cao, J.D.: Novel bifurcation results for a delayed fractional-order quaternion-valued neural network. Neural Netw. 117, 67-93 (2019).

13. Huang, C.D., Zhao, X., Wang, X.H., Wang, Z.X., Xiao, M., Cao, J.D.: Disparate delays-induced bifurcations in a fractional-order neural network. J. Franklin Inst. 356(5), 2825-2846 (2019)

14. Li, Y., Li, C.D.: Matrix measure strategies for stabilization and synchronization of delayed BAM neural networks. Nonlinear Dyn. 84(3), 1759-1770 (2016) 
15. Aghababa, M.P.: Synchronization and stabilization of fractional second-order nonlinear complex systems. Nonlinear Dyn. 80(4), 1731-1744 (2015)

16. Zhang, J.E.: Linear-type discontinuous control of fixed-deviation stabilization and synchronization for fractional-order neurodynamic systems with communication delays. IEEE Access 6, 52570-52581 (2018)

17. Zhou, Y.H., Li, C.D., Huang, T.W., Wang, X.: Impulsive stabilization and synchronization of Hopfield-type neural networks with impulse time window. Neural Comput. Appl. 28(4), 775-782 (2017)

18. Tan, M.C., Tian, W.X.: Finite-time stabilization and synchronization of complex dynamical networks with nonidentical nodes of different dimensions. Nonlinear Dyn. 79(1), 731-741 (2015)

19. Wu, A.L., Zeng, Z.G.: Global Mittag-Leffler stabilization of fractional-order memristive neural networks. IEEE Trans. Neural Netw. Learn. Syst. 28(1), 206-217 (2017)

20. Yang, H.J., Liu, J.K.: An adaptive RBF neural network control method for a class of nonlinear systems. IEEE/CAA J. Autom. Sin. 5(2), 457-462 (2018)

21. Tan, M.C., Pan, Q., Zhou, X.: Adaptive stabilization and synchronization of non-diffusively coupled complex networks with nonidentical nodes of different dimensions. Nonlinear Dyn. 85(1), 303-316 (2016)

22. Ma, W.Y., Li, C.P., Wu, Y.J., Wu, Y.Q.: Adaptive synchronization of fractional neural networks with unknown parameters and time delays. Entropy 16(12), 6286-6299 (2014)

23. Huang, C.D., Li, H., Cao, J.D.: A novel strategy of bifurcation control for a delayed fractional predator-prey model. Appl. Math. Comput. 347, 808-838 (2019)

24. Tan, X.G., Cao, J.D., Li, X.D.: Consensus of leader-following multiagent systems: a distributed event-triggered impulsive control strategy. IEEE Trans. Cybern. 49(3), 792-801 (2019)

\section{Submit your manuscript to a SpringerOpen ${ }^{\circ}$ journal and benefit from:}

- Convenient online submission

- Rigorous peer review

- Open access: articles freely available online

- High visibility within the field

- Retaining the copyright to your article

Submit your next manuscript at $\gg$ springeropen.com 